\title{
Observation of macroscopic valley-polarized monolayer exciton-polaritons at room temperature
}

\author{
N. Lundt, ${ }^{1, *}$ S. Stoll,,${ }^{1}$ P. Nagler, ${ }^{2}$ A. Nalitov, ${ }^{3}$ S. Klembt, ${ }^{1}$ S. Betzold,${ }^{1}$ J. Goddard, ${ }^{1}$ E. Frieling, ${ }^{1}$ A. V. Kavokin, ${ }^{3,4,5}$ \\ C. Schüller, ${ }^{2}$ T. Korn, ${ }^{2}$ S. Höfling, ${ }^{1,6}$ and C. Schneider ${ }^{1, *}$ \\ ${ }^{1}$ Technische Physik and Wilhelm-Conrad-Röntgen-Research Center for Complex Material Systems, \\ Universität Würzburg, D-97074 Würzburg, Am Hubland, Germany \\ ${ }^{2}$ Department of Physics, University of Regensburg, Regensburg D-93040, Germany \\ ${ }^{3}$ Physics and Astronomy School, University of Southampton, Highfield, Southampton SO171BJ, United Kingdom \\ ${ }^{4}$ SPIN-CNR, Viale del Politecnico 1, I-00133 Rome, Italy \\ ${ }^{5}$ Russian Quantum Center, 100, Novaya str., Skolkovo, Moscow region, Russia \\ ${ }^{6}$ School of Physics and Astronomy, University of St. Andrews, St. Andrews KY 16 9SS, United Kingdom \\ (Received 5 July 2017; revised manuscript received 6 November 2017; published 5 December 2017)
}

\begin{abstract}
In this Rapid Communication, we address the chiral properties of valley exciton-polaritons in a monolayer of $\mathrm{WS}_{2}$ in the regime of strong light-matter coupling with a Tamm-plasmon resonance. We observe that the effect of valley polarization, which manifests in the circular polarization of the emitted photoluminescence as the sample is driven by a circularly polarized laser, is strongly enhanced in comparison to bare $\mathrm{WS}_{2}$ monolayers and can even be observed under strongly nonresonant excitation at ambient conditions. In order to explain this effect in more detail, we study the relaxation and decay dynamics of exciton-polaritons in our device, elaborate the role of the dark state, and present a microscopic model to explain the wave-vector-dependent valley depolarization by the linear polarization splitting inherent to the microcavity. We believe that our findings are crucial for designing novel polariton-valleytronic devices which can be operated at room temperature.
\end{abstract}

DOI: 10.1103/PhysRevB.96.241403

Introduction. Excitons which are hosted in two-dimensional atomic crystals of transition-metal dichalcogenides (TMDCs) have a variety of intriguing optical properties. This includes their enormous exciton binding energies up to $550 \mathrm{meV}[1,2]$, their ultrafast dipole transitions [3], as well as the materials' unique spin- and valley-related properties [4-7]. The latter are a direct consequence of the broken inversion symmetry of the monolayers in combination with a strong spin-orbit coupling inherited by the transition-metal atoms, lifting the polarization degeneracy of the high-symmetry $K$ points at the corners of the Brillouin zone. Thus, excitons at the $K$ and $K^{\prime}$ points are tagged with a valley index, or valley pseudospin, which is more robust with respect to depolarization than the exciton pseudospin in conventional III-V semiconductors. The coupling of spin and valley degrees of freedom in TMDC monolayers, in principle, allows to build electronic and optoelectronic devices based on rich polarization and spin features, giving rise to the field of valleytronics [7]. Recently, it has been shown that cavity effects can enhance the time-averaged degree of exciton and trion valley polarizations [8-11], which is partly explained by a speedup of the relaxation dynamics of trions for the case of trion-polaritons at cryogenic temperatures [8]. However, in previous experiments carried out at ambient conditions [9-11], no dynamic data were provided. Moreover, the role of the dark exciton state, which is considered crucial for a high degree of valley polarization in tungsten-based TMDCs [12], has not been elaborated in detail. In addition to the electron-hole exchange interaction, the artificial magnetic field provided by the polarization splitting of the cavity resonances acts as a depolarization mechanism which has to be taken into account. Here, we investigate the

\footnotetext{
*Corresponding authors: nils.lundt@physik.uni-wuerzburg.de; christian.schneider@physik.uni-wuerzburg.de
}

relaxation and valley depolarization of valley-tagged excitonpolaritons evolving in a Tamm-plasmon structure $[13,14]$ with an integrated monolayer of $\mathrm{WS}_{2}$ at ambient conditions. Time-resolved photoluminescence measurements were carried out to shed more light onto the relaxation dynamics of the observed exciton-polaritons. We discuss the role of the interplay between dark and bright states, which is affected by a dramatic change in the bright-state dispersion relation in the polariton framework. Finally, we present a theoretical model of the depolarization mechanism along the dispersion relation, which explains the measured distinct polarization dependence on the in-plane wave vector.

Monolayer characterization. The investigated $\mathrm{WS}_{2}$ monolayer was mechanically exfoliated from a bulk crystal and subsequently transferred onto a distributed Bragg reflector (DBR) with a viscoelastic polymer stamp. The monolayer was identified by its optical contrast and its distinct photoluminescence (PL). The DBR is composed of ten $\mathrm{SiO}_{2} / \mathrm{TiO}_{2}$ layers with thicknesses of $105 \mathrm{~nm} / 65 \mathrm{~nm}$, respectively. The photoluminescence of the monolayer (on top of the DBR), presented in Fig. 1(a), was recorded under 568-nm excitation [continuous-wave (cw) laser (Coherent sapphire), $0.7 \mathrm{~mW}$ ] and ambient conditions. The spectrum shows a clear resonance at $618.57 \mathrm{~nm} / 2.004 \mathrm{eV}$ with a linewidth (FWHM) of $29 \mathrm{meV}$, which we attribute to the neutral excitonic transition of the $A$ excitons. The low-energy shoulder of the peak can be assigned to a trionic transition with an exciton-trion energy splitting of $42 \mathrm{meV}$, which strongly grows in intensity at lower sample temperatures, in line with previous observations [15]. First, we study the polarization properties of our bare monolayer as a function of the detuning of the pump laser and temperature. Figure 1(a) depicts a polarization-resolved photoluminescence spectrum of the monolayer exciton recorded at $290 \mathrm{~K}$. The sample was excited with a $\sigma^{+}$-polarized laser $(568 \mathrm{~nm})$, and the luminescence is detected in $\sigma^{+} / \sigma^{-}$configurations. 
(a)

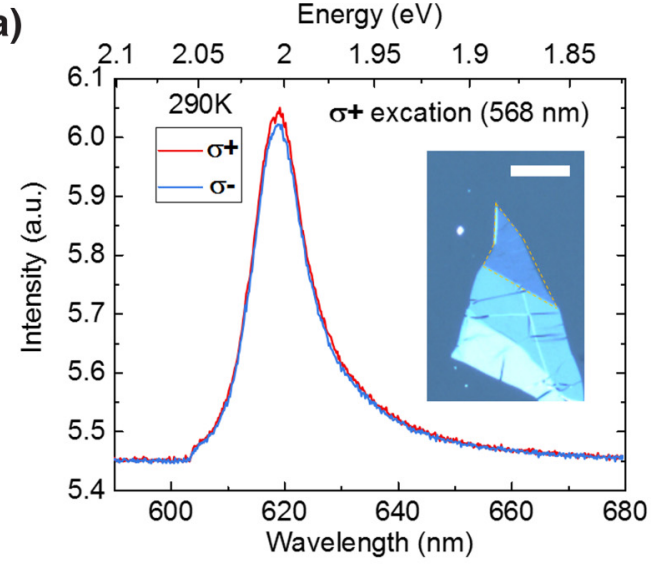

(b)

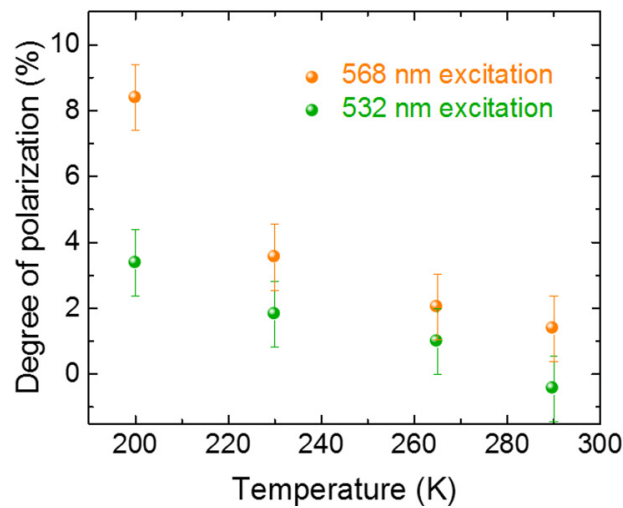

FIG. 1. Characterization of a $\mathrm{WS}_{2}$ monolayer. (a) PL spectra of a monolayer (shown and marked by yellow lines in the inset where the scale bar corresponds to $30 \mu \mathrm{m}$ ) at $290 \mathrm{~K}$ under $\sigma^{+}$-polarized excitation at $568 \mathrm{~nm}$ recorded in $\sigma^{+} / \sigma^{-}$configurations. (b) Degree of circular polarization as a function of temperature for 532- and 568-nm excitation wavelengths.

The degree of circular polarization (DOCP) is calculated via $P=\frac{I\left(\sigma^{+}\right)-I\left(\sigma^{-}\right)}{I\left(\sigma^{+}\right)+I\left(\sigma^{-}\right)}$, where $I$ is the integrated PL intensity. The degree of circular polarization of the emitted light as a function of temperature and excitation energy (532-nm vs 568-nm excitation) is plotted in Fig. 1(b). Although the increase in $P$ with decreasing temperature and an excitation energy closer to the exciton resonance is in excellent agreement with previous findings [16], we emphasize that no significant DOCP can be extracted at ambient conditions.

Exciton-polaritons. The photonic Tamm structure is completed by capping the $\mathrm{WS}_{2}$ monolayer by $70 \mathrm{~nm}$ of polymethylmethacrylate (PMMA) and evaporation of $40 \mathrm{~nm}$ of silver on top of the PMMA layer [Fig. 2(a)]. A final $\mathrm{SiO}_{2}$ layer was deposited to protect the silver layer from degradation. The bottom DBR supports a very high reflectivity of $99.97 \%$ in a spectral range between 540 and $680 \mathrm{~nm}$, and the photonic microstructure features a strong field enhancement close to the metallic interface [see Fig. 2(b)] at the monolayer location. This strong field enhancement makes such structures particularly interesting to study the physics of excitonpolaritons in solid-state systems [17]. The layer thicknesses, illustrated in Fig. 2(b) as the sequence of the corresponding refractive indices, were designed to promote an optical mode energetically close to the exciton resonance and to spatially overlap with the monolayer. The resonance of the empty cavity was probed in a white-light reflectivity measurement presented in Fig. 2(c) and features a quality factor of 120.

The formation of room-temperature exciton-polaritons in our device is confirmed by single-shot angle-resolved photoluminescence measurements in a back-Fourier plane imaging configuration (see the Supplemental Material [18]). Utilizing a high magnification $(50 \times)$ microscope objective with a numerical aperture of 0.65 allows us to project an in-plane momentum range of up to $5.5 \mu \mathrm{m}^{-1}$ onto the CCD chip of our spectrometer in this imaging configuration [8]. For polarization measurements we use a combination of a linear polarizer and a $\lambda / 4$ wave plate to generate $\left(\sigma^{+} / \sigma^{-}\right)$-polarized light and analyzed the emitted signal with a rotatable $\lambda / 4$ wave plate followed by a linear polarizer. We used a nonpolarizing beam splitter preserving $s$ and $p$ components by $98 \%$. In addition, the incident laser was analyzed by a polarimeter at various positions in the optical path, and the $\lambda / 4$ wave plate was corrected slightly in order to compensate for the loss in degree of circular polarization at the beam splitter. This ensures a circular degree of polarization of more than $99.9 \%$. The emitted light on the detection path is still subject to a relative error of $2 \%$. The main error contribution is the laser power fluctuation on the order of $1 \%$.

The luminescence which we collect from our device is depicted in Fig. 2(d). It features the typical distinct dispersion relation of the lower branch of cavity exciton-polaritons, which emerge in the strong-coupling regime between excitons and cavity photons. At low- $k$ values, this dispersion is dominated by the low mass [19] of two-dimensional cavity photons (approximately $10^{-5} m_{\mathrm{e}}, m_{\mathrm{e}}$ being the free-electron mass), whereas its curvature features an inflection point at $k \sim$ $3.0 \mu \mathrm{m}^{-1}$. We can fit the dispersion with a two-coupled oscillator model [17] to extract the Rabi splitting and the excitonphoton detuning of our device. Here, we take advantage of the fact that we can observe the empty cavity dispersion as a faint photoluminescence branch in the background. This PL stems from edge regions of the monolayer and is weakly coupled to the cavity mode. The fit yields a Rabi splitting as large as $80 \mathrm{meV}$ and an exciton-photon detuning of $-55 \mathrm{meV}$ at $k=0$. The observed coupling strength is in good agreement with previous findings on strongly coupled $\mathrm{WS}_{2}$ monolayers in a microcavity considering a comparable mode volume [20]. The upper polariton branch cannot be observed due to its very low population resulting from the large normal mode coupling strength.

We note that, due to the large Rabi splitting, our polariton dispersion crosses both the (weakly coupled) trion resonance as well as the dark exciton ( $55 \mathrm{meV}$ below the exciton) [21]. Both resonances are indicated by arrows in Fig. 2(d). Neither the trion nor the dark-state resonance are expected to carry enough oscillator strength at room temperature in order to strongly couple with the cavity mode. The small oscillator strength seems plausible since the resonances cannot be measured in absorption experiments at room temperature. Since the polariton bright state now is favored energetically and 
(a)

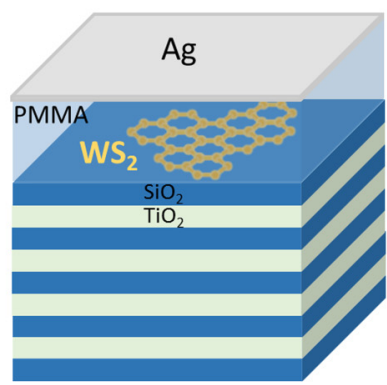

(c)

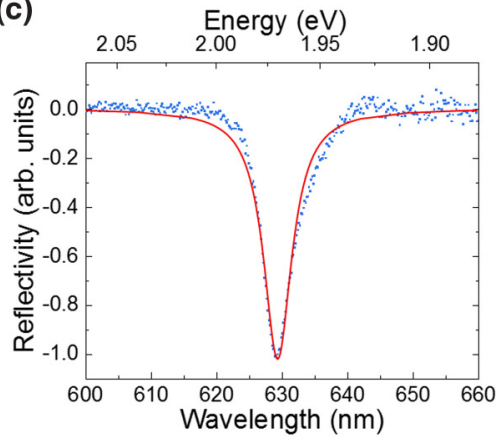

(b)

(d)
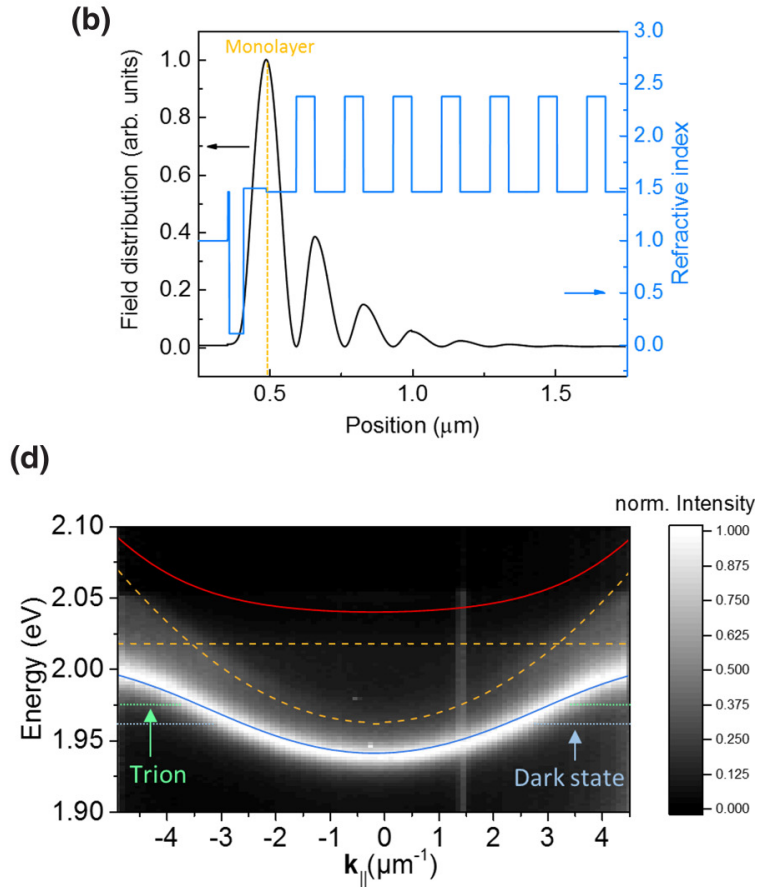

FIG. 2. (a) schematic of the photonic structure with the integrated $\mathrm{WS}_{2}$ monolayer. (b) Layer sequence of the photonic structure illustrated by the corresponding refractive indices and the calculated optical field distribution within the photonic structure. The position of the monolayer in the photonic structure is marked as the orange dashed line. (c) Measured reflectivity spectrum of the empty cavity at the zero in-plane wave vector. The linewidth corresponds to a quality factor of 120. (d) Exciton-polariton dispersion relation including a coupled oscillator fit. The upper and lower polariton branches are drawn in red and blue, respectively, where the uncoupled exciton and cavity modes are indicated by the orange dashed lines. Expected energies of the trion and the dark state are indicated in the background.

carries significantly more oscillator strength, the PL intensity of the polariton branch governs the dispersion. However, since both resonances are in the perturbative regime with our cavity resonance, weak-coupling conditions can yield a transfer of populations to the polariton states.

Exciton and polariton dynamics. The fitting procedure also provides the light-matter coupling strength, which in turn can be used to assess the radiative lifetime of the $\mathrm{WS}_{2}$ valley excitons. The Rabi splitting yields a direct connection with the exciton oscillator strength and the effective cavity length [22], which can be expressed as

$$
\Omega_{R}=2 * \sqrt{\frac{2 \Gamma_{0} c}{n_{c}\left(L_{\mathrm{DBR}}+L_{C}\right)}} .
$$

Here, $\Gamma_{0}$ represents the radiative decay of the excitons, $n_{c}$ is the effective index of the cavity, and $L_{\mathrm{DBR}}+L_{C}$ is the effective cavity length. $c$ is the speed of light in free space. Introducing our system parameters (see the Supplemental Material [23]), we determine a radiative decay time as short as $220 \mathrm{fs}$. We note that this decay time is in good agreement with previous experimental studies on $\mathrm{WSe}_{2}$ monolayers [3] and theoretical predictions [24]. In our case, we emphasize that our method allows us to solely probe the radiative decay time as the nonradiative decay channels would not contribute to the light-matter coupling strength. Nevertheless, taking the radiative exciton lifetime as an input, a calculation of the lower polariton radiative lifetimes in our strongly coupled system yields values that are three to nine times shorter (see the Supplemental Material [25]).

In order to assess the full relaxation dynamics of our system, we perform streak-camera measurements on the bare $\mathrm{WS}_{2}$ monolayer as well as on the fully built cavity in the strong-coupling regime. For this, the sample was excited by a frequency-doubled pulsed fiber laser system [TOPTICA TVIS, pulse length (FWHM) $180 \mathrm{fs,} \mathrm{pulse} \mathrm{repetition} \mathrm{rate} 80 \mathrm{MHz}$ ] tuned to a central wavelength of $568 \mathrm{~nm}$, coupled into a $100 \times$ microscope objective, and focused to a spot diameter of less than $1 \mu \mathrm{m}$ on the sample surface. The PL from the sample was collected using the same objective and coupled into a grating spectrometer where it was detected using a streak camera coupled to the spectrometer and electronically synchronized with the pulsed laser system. The temporal resolution of this setup (HWHM of the pulsed laser trace) is below 4 ps (see the Supplemental Material [18]).

Figure 3(a) depicts the photoluminescence decay curves of polariton states at high in-plane $k$ vectors $\left(\sim 5 \mu \mathrm{m}^{-1}\right)$, often referred to as the reservoir, and of the polariton ground state $\left(k=0 \mu \mathrm{m}^{-1}\right)$. Both time traces exhibit two dominant exponential decay channels and a very weak third decay. The reservoir decay time constants $\tau_{1}$ and $\tau_{2}$ of 8.8 and $30.3 \mathrm{ps,}$ respectively, are in excellent agreement with the reference measurement on the bare $\mathrm{WS}_{2}$ monolayer $(8.2$ and $31.6 \mathrm{ps}$, respectively (see the Supplemental Material [26]). This is well in line with the highly excitonic character of the polaritons at high- $k$ vectors and previous findings [27,28]. This also supports the idea that the scatterings outside the light cone 

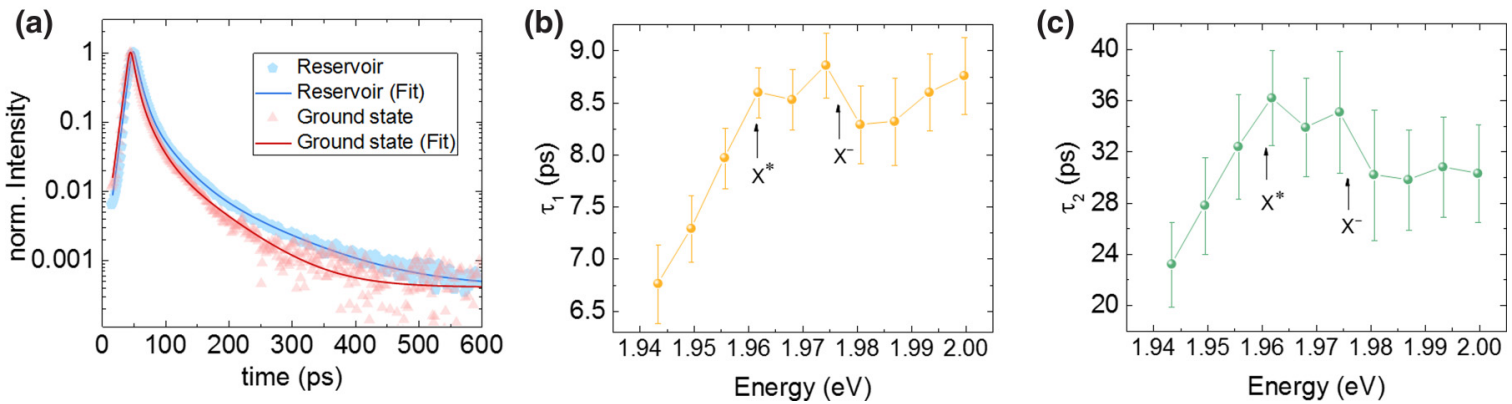

FIG. 3. Relaxation dynamics. (a) Decay curves of the polariton reservoir $\left(\sim 5 \mu \mathrm{m}^{-1}\right)$ and the polariton ground state $\left(0 \mu \mathrm{m}^{-1}\right)$. (b) Time constant $\tau_{1}$ as a function of emission energy. (c) Time constant $\tau_{2}$ as a function of emission energy. Expected energies of the dark-state $X^{*}$ and trion $X^{-}$are indicated by black arrows.

and close to the light cone edge are not significantly affected. In contrast, the characteristic time constants decrease to 6.8 and $23.2 \mathrm{ps}$, respectively, in the polariton ground states. The third decay channel only makes up a minor fraction of the PL intensity $(<1 \%)$ and is on the order of 100 ps in all measurements. In more detail, Figs. 3(b) and 3(c) illustrate the decay constants $\tau_{1}$ and $\tau_{2}$ as a function of energy which is related to the in-plane wave vector through the dispersion relation. Here, the low-energy end at $1.94 \mathrm{eV}$ represents the polariton ground state, whereas the high-energy tail at $2.01 \mathrm{eV}$ is attributed to emission from the highly excitonic reservoir.

Interestingly, both time constants feature a decrease towards lower energies as well as a slight increase between 1.96 and $1.975 \mathrm{eV}$. In fact, this increase occurs at energies where the polariton dispersion crosses the dark state and the trion resonances, which feature slower decay dynamics [29]. This indicates an indirect pumping mechanism from these weakly coupled states into the polariton states.

Because of the very fast radiative decay of the exciton and polariton states inside the light cone, we attribute the decay times as the depopulation dynamics of the original states from which excitons-polaritons scatter into the measured state. These states presumably lie outside the light cone or can be attributed to trion or dark states. Here, we attribute the first channel to fast phonon-assisted carrier relaxation from outside of the light cone (followed by fast radiative decay), whereas the second slower decay could be an indication for a transfer from the dark exciton state.

Polariton-valley polarization. In the following, we address the spin- and valley-related properties of our polariton system via polarization-resolved spectroscopy. Here, we inject reservoir excitons nonresonantly via circularly polarized pump lasers with wavelengths of 532 or $568 \mathrm{~nm}$, respectively, and measure the DOCP as a function of the polariton wave vector. The results of this experiment, shown in Fig. 4(a) (532-nm excitation) and Fig. 4(b) (568-nm excitation), are strikingly different from the case of the bare monolayer where the DOCP is marginal at room temperature. For the case of the 568-nm-excitation wavelength, we observe a DOCP as large as $10 \%$ from the polariton ground state at ambient conditions. These findings are well in line with very recent results [11]. We furthermore observe a distinct dependency of the DOCP on the in-plane wave vector. The observation of this high circular polarization from the polariton states is, at first sight, surprising since the system follows the dynamics of the bright exciton reservoir, which manifest in the comparable scattering dynamics for the bare monolayer and the polariton at high wave vectors and which we consider as strongly depolarized at $300 \mathrm{~K}$ (see Fig. 1).

Whereas the relaxation into the bright exciton/excitonpolariton states is almost completely depolarizing, the dark exciton state is considered to maintain the excitation polarization [12,30] as illustrated in Fig. 4(c). At zero in-plane wave vector, the dark exciton has no oscillator strength, yet but it significantly accumulates oscillator strength at high in-plane momenta [21]. Hence, it is reasonable to assume that it can weakly couple to corresponding high- $k$ polariton states [illustrated in Fig. 4(c)], followed by a radiative decay or a redistribution along the polariton branch. The coupling to the bright state is enhanced strongly by the cavity effect since emitted light from the dark state can be reabsorbed by the bright polariton state. In fact, the emission of the dark state should be enhanced when weakly coupled to the cavity mode, whereas the absorption by the bright polariton state is more likely since it should carry significantly more oscillator strength even at high wave vectors. Thus, we determine a second reservoir at large $k_{\|}$, which is robust against valley depolarization since dark excitons are not subject to strong exchange interactions [12]. (See the Supplemental Material [31] for further details on the transfer from a dark to a bright state).

The transfer from the dark state can be followed by a redistribution of polaritons along the polariton branch. However, in this case the polarized polaritons are again subject to the strong exchange interactions, which can lead to valley depolarization during their redistribution. In the following, we provide a description and an analytic expression of the valley depolarization in our polariton system. Here, we consider a gas of free polaritons with parabolic dispersion. We will account for: (1) acoustic phonon-assisted energy relaxation, which is dominant below the condensation threshold and (2) MaialleSilva-Sham-type spin relaxation stemming from the interplay of a momentum-dependent effective magnetic field acting on the polariton pseudospin and stochastic elastic momentum scattering. The dynamics of spin relaxation in a polariton gas has been modeled numerically by solving the full set of Boltzmann kinetic equations [32]. In contrast, here we opted for a simplified analytical model with a very limited number of free parameters. We are interested in the analytical expression 
(a)

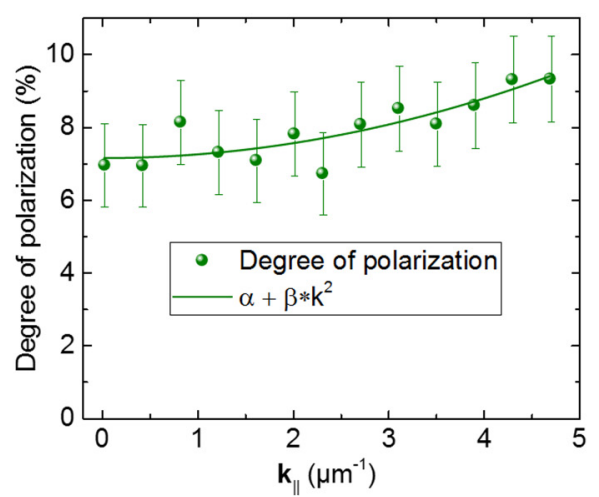

(b)

\section{$568 \mathrm{~nm}$ excitation}

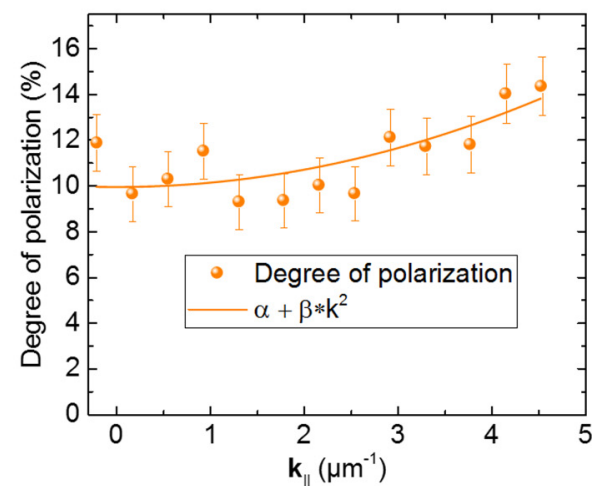

(c)

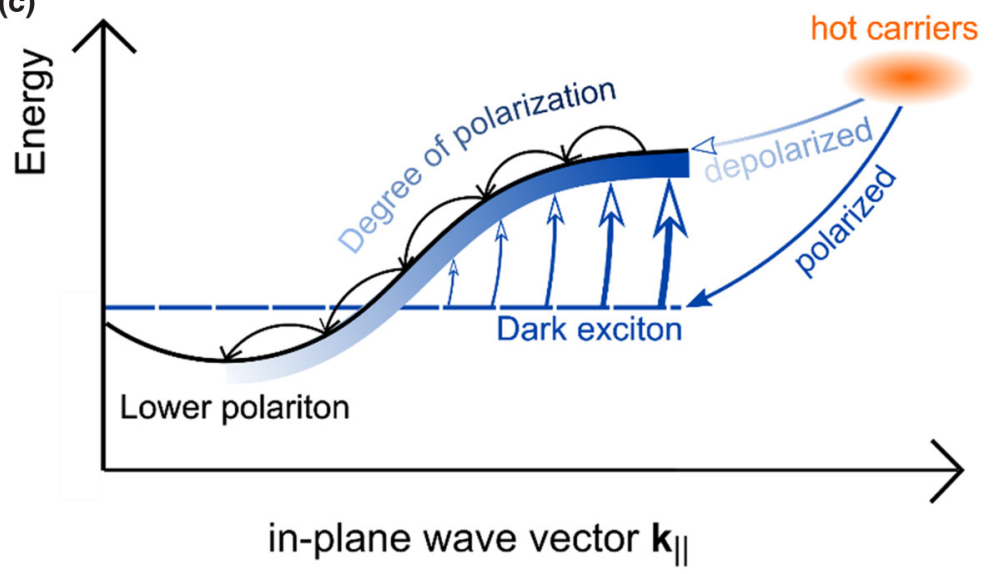

FIG. 4. Polariton-valley polarization, (a) degree of circular polarization as a function of the in-plane wave vector under 532-nm excitation. (b) Degree of circular polarization as a function of the in-plane wave vector under 568-nm excitation. (c) The schematic model for polariton-valley polarization.

for the circular polarization of the polariton emission as a function of the in-plane momentum for the stationary state corresponding to the $\mathrm{cw}$ pumping regime.

We discretize the continuous problem and reformulate it in terms of a ladder of discrete quantum states where direct transitions are allowed only between the neighboring energy levels. We consider phonon-assisted inelastic transitions for a polariton with the momentum $\mathbf{p}$ and neglected nonlinear effects caused by exciton-exciton scattering. In contrast to quantum-well-based microcavities where the main contribution to phonon-assisted exciton energy relaxation stems from the phonons with momenta oriented along the growth direction [33], the TMDC atomic layers are only coupled to the substrate by the van der Waals force so that the excitons only emit in-plane phonons [34]. The characteristic energy lost in a single act of scattering that is taken as an interlevel distance in our model then is derived from the energy and momentum conservation rules. The rates of polariton transitions between the levels $\alpha_{i}=\alpha p_{i}^{3}$ depend on the wave vector via the excitonphonon matrix element, the exciton Hopfield coefficient, and the density of the polariton states.

The spin-relaxation rate $\gamma_{i}$ is governed by the MaialleSilva-Sham mechanism $\gamma_{i}=\Omega_{i}^{2} \tau$, where $\Omega_{i}$ is the value of the effective magnetic field and $\tau$ is the polariton transport time, which we assume as constant. The effective field $\Omega_{i}$ originates from the TE-TM splitting of the photonic-polariton component and the long-range part of the electron-hole exchange acting on the excitonic component. In the vicinity of the polariton dispersion minimum the photonic component is dominant, hence, the effective field is given by the TE-TM splitting, which is quadratic in polariton momentum, allowing us to assume $\gamma_{i}=\beta p_{i}^{4}$.

This yields an analytical expression for the DOCP as a function of the momentum which reads

$$
P(p)=P(0)+\frac{\beta}{2 \alpha} p^{2} .
$$

As shown in Figs. 4(a) and 4(b), our data can be well fitted by this expression, which confirms our initial assumptions about the important effect of TE-TM splitting in our cavity on the spin depolarization of valley excitons in TMDC-based cavities.

Although our model succeeds to fit the experimental data very well, we note that direct polariton-polariton scattering from reservoir states must be taken into consideration in particular for large pump powers where exciton-exciton scattering and bosonic stimulation become important. This process would result in a speedup of the relaxation dynamics, which is in fact observed for the ground state. 
Conclusion. In conclusion, our Rapid Communication sheds light onto the interplay among relaxation, depolarization, and the decay of valley exciton-polaritons. First, we demonstrate the formation of exciton-polaritons in a photonic microstructure with excitons in a $\mathrm{WS}_{2}$ monolayer with a light-matter coupling strength of $80 \mathrm{meV}$, corresponding to a purely radiative decay time of excitons as short as $220 \mathrm{fs}$. We find that the polariton scattering dynamics at a high wave vector and close to the light cone edge are not affected strongly as compared to the bare monolayer, yet relaxation of the polaritons towards the ground state is slightly enhanced even at modest densities. Despite the slow relaxation, we map out a significant enhancement of polaritonic-valley polarization up to $14 \%$ at high wave vectors. We attribute this increase to the cavity-enhanced transfer from dark exciton states to the polariton branch, which is more effective at high wave vectors. The polarization relaxation is explained within a microscopic model, which accounts for energy and spin relaxation of exciton-polaritons in the presence of the linear polarization splitting of the microcavity. We believe that our findings represent a major step towards harnessing spin-valley coupling in light-matter-coupled devices operated at room temperature.

Acknowledgments. This work has been supported by the State of Bavaria, the ERC (unlimit-2D), as well as the DFG via Grants No. SFB689, No. GRK 1570, and No. KO3612/1-1. We thank S. Tongay for supporting this project. A.K. acknowledges partial financial support from the Ministry of Education and Science of the Russian Federation in the framework of increase Competitiveness Program of NUST "MISIS", No, 211, implemented by a governmental decree.
[1] M. M. Ugeda et al., Giant bandgap renormalization and excitonic effects in a monolayer transition metal dichalcogenide semiconductor, Nat. Mater. 13, 1091 (2014).

[2] A. Chernikov, T. C. Berkelbach, H. M. Hill, A. Rigosi, Y. Li, O. B. Aslan, D. R. Reichman, M. S. Hybertsen, and T. F. Heinz, Exciton Binding Energy and Nonhydrogenic Rydberg Series in Monolayer $\mathrm{WS}_{2}$, Phys. Rev. Lett. 113, 076802 (2014).

[3] C. Poellmann et al., Resonant internal quantum transitions and femtosecond radiative decay of excitons in monolayer $\mathrm{WSe}_{2}$, Nat. Mater. 14, 889 (2015).

[4] D. Xiao, G.-B. Liu, W. Feng, X. Xu, and W. Yao, Coupled Spin and Valley Physics in Monolayers of $\mathrm{MoS}_{2}$ and Other Group-VI Dichalcogenides, Phys. Rev. Lett. 108, 196802 (2012).

[5] Q. H. Wang, K. Kalantar-Zadeh, A. Kis, J. N. Coleman, and M. S. Strano, Electronics and optoelectronics of two-dimensional transition metal dichalcogenides, Nat. Nanotechnol. 7, 699 (2012).

[6] L. Cao, Two-dimensional transition-metal dichalcogenide materials: Toward an age of atomic-scale photonics, MRS Bull. 40, 592 (2015).

[7] K. F. Mak and J. Shan, Photonics and optoelectronics of 2D semiconductor transition metal dichalcogenides, Nat. Photonics 10, 216 (2016).

[8] N. Lundt et al., Valley polarized relaxation and upconversion luminescence from Tamm-Plasmon Trion-Polaritons with a $\mathrm{MoS}_{2}$ monolayer, 2D Mater. 4, 025096 (2017).

[9] Y. Chen, J. D. Cain, T. K. Stanev, V. P. Dravid, and P. Nathaniel, Valley-Polarized Microcavity Exciton-Polaritons in a Monolayer Semiconductor, Nat. Photonics 11, 431 (2017).

[10] S. Dufferwiel et al., Valley-addressable polaritons in atomically thin semiconductors, Nat. Photonics 11, 497 (2017).

[11] Z. Sun et al., Optical control of room-temperature valley polaritons, Nat. Photonics 11, 491 (2017).

[12] M. Baranowski, A. Surrente, D. K. Maude, M. Ballottin, A. A. Mitioglu, P. C. M. Christianen, Y. C. Kung, D. Dumcenco, A. Kis, and P. Plochocka, Dark excitons and the elusive valley polarization in transition metal dichalcogenides, 2D Mater. 4, 025016 (2017).

[13] N. Lundt et al., Room temperature Tamm-Plasmon ExcitonPolaritons with a $\mathrm{WSe}_{2}$ monolayer, Nat. Commun. 7, 13328 (2016).
[14] T. Hu et al., Strong coupling between Tamm plasmon polariton and two dimensional semiconductor excitons, Appl. Phys. Lett. 110, 051101 (2017).

[15] G. Plechinger et al., Identification of excitons, trions and biexcitons in single-layer $\mathrm{WS}_{2}$, Phys. Status Solidi Rapid Res. Lett. 9, 457 (2015)

[16] A. T. Hanbicki et al., Anomalous temperature-dependent spinvalley polarization in monolayer $\mathrm{WS}_{2}$, Sci. Rep. 6, 18885 (2016).

[17] H. Deng, H. Haug, and Y. Yamamoto, Exciton-polariton BoseEinstein condensation, Rev. Mod. Phys. 82, 1489 (2010).

[18] See Supplemental Material at http://link.aps.org/supplemental/ 10.1103/PhysRevB.96.241403 for a schematic illustration of the setup.

[19] M. Richard, The not-so-effective mass of photons in a planar optical cavity, arXiv:1512.01130.

[20] L. C. Flatten et al., Room-temperature exciton-polaritons with two-dimensional $\mathrm{WS}_{2}$, Sci. Rep. 6, 33134 (2016).

[21] G. Wang, C. Robert, M. M. Glazov, F. Cadiz, E. Courtade, T. Amand, D. Lagarde, T. Taniguchi, K. Watanabe, B. Urbaszek, and X. Marie, In-Plane Propagation of Light in Transition Metal Dichalcogenide Monolayers: Optical Selection Rules, Phys. Rev. Lett. 119, 047401 (2017).

[22] V. Savona, L. C. Andreani, P. Schwendimann, and A. Quattropani, Quantum well excitons in semiconductor microcavities: Unified treatment of weak and strong coupling regimes, Solid State Commun. 93, 733 (1995).

[23] See Supplemental Material at http://link.aps.org/supplemental/ 10.1103/PhysRevB.96.241403 for details on the system parameters.

[24] M. Palummo, M. Bernardi, and J. C. Grossman, Exciton radiative lifetimes in two-dimensional transition metal dichalcogenides, Nano Lett. 15, 2794 (2015).

[25] See Supplemental Material at http://link.aps.org/supplemental/ 10.1103/PhysRevB.96.241403 for calculations on the radiative polariton lifetime.

[26] See Supplemental Material at http://link.aps.org/supplemental/ 10.1103/PhysRevB.96.241403 for the reference measurement.

[27] M. Perrin et al., Relaxation dynamics of microcavity polaritons in the presence of an electron gas, Phys. Status Solidi C 2, 3920 (2005). 
[28] P. Senellart, J. Bloch, B. Sermage, and J. Y. Marzin, Microcavity polariton depopulation as evidence for stimulated scattering, Phys. Rev. B 62, R16263(R) (2000).

[29] G. Wang, L. Bouet, D. Lagarde, M. Vidal, A. Balocchi, T. Amand, X. Marie, and B. Urbaszek, Valley dynamics probed through charged and neutral exciton emission in monolayer $\mathrm{WS}_{2}$, Phys. Rev. B 90, 075413 (2014).

[30] G. Plechinger et al., Trion fine structure and coupled spin-valley dynamics in monolayer tungsten disulfide, Nat. Commun. 7, 12715 (2016).

[31] See Supplemental Material at http://link.aps.org/supplemental/ 10.1103/PhysRevB.96.241403 for dark-state transfer calculations.
[32] K. V. Kavokin, I. A. Shelykh, A. V. Kavokin, G. Malpuech, and P. Bigenwald, Quantum Theory of Spin Dynamics of Exciton-Polaritons in Microcavities, Phys. Rev. Lett. 92, 017401 (2004).

[33] C. Piermarocchi, F. Tassone, V. Savona, A. Quattropani, and P. Schwendimann, Nonequilibrium dynamics of free quantum-well excitons in time-resolved photoluminescence, Phys. Rev. B 53, 15834 (1996).

[34] A. O. Slobodeniuk and D. M. Basko, Spin-flip processes and radiative decay of dark intravalley excitons in transition metal dichalcogenide monolayers, 2D Mater. 3, 035009 (2016). 